\title{
PATACSDB - The database of polyA translational attenuators in coding sequences
}

Malgorzata Habich, Sergej Djuranovic, Pawel Szczesny

Recent addition to the repertoire of gene expression regulatory mechanisms are polyadenylate (polyA) tracks encoding for poly-lysine runs in protein sequences. Such tracks stall translation apparatus and induce frameshifting independently of the effects of charged nascent poly-lysine sequence on the ribosome exit channel. As such they substantially influence the stability of mRNA and amount of protein produced from a given transcript. Single base changes in these regions are enough to exert a measurable response on both protein and mRNA abundance, and makes each of these sequences potentially interesting case studies for effects of synonymous mutation, gene dosage balance and natural frameshifting. Here we present the PATACSDB, a resource that contain comprehensive list of polyA tracks from over 250 eukaryotic genomes. Our data is based on Ensembl genomic database of coding sequences and filtered with algorithm of $12 \mathrm{~A}-1$ which selects sequences of polyA tracks with a minimal length of $12 \mathrm{~A}$ 's allowing for one mismatched base. The PATACSDB database is accesible at:

http://sysbio.ibb.waw.pl/patacsdb. Source code is available for download from GitHub repository at http://github.com/habich/PATACSDB, including the scripts to recreate the database from scratch on the user's own computer. 
1 PATACSDB - The Database of PolyA

\section{Translational Attenuators in Coding}

\section{${ }_{3}$ Sequences}

4

5 Malgorzata Habich ${ }^{1}$, Sergej Djuranovic ${ }^{2}$, Pawel Szczesny ${ }^{1,3 \#}$

6

71 Institute of Biochemistry and Biophysics Polish Academy of Sciences, Department of Bioinformatics,

8 Pawinskiego 5a, 02-106 Warsaw, Poland

92 Washington University School of Medicine, Department of Cell Biology and Physiology, St. Louis,

10 MO63110, USA

113 Faculty of Biology, Institute of Experimental Plant Biology and Biotechnology, University of Warsaw,

12 ul. Miecznikowa 1, 02-096 Warsaw, Poland

15 \# Correspondence should be addressed to:

16 Pawel Szczesny

17 Department of Bioinformatics

18 Institute of Biochemistry and Biophysics Polish Academy of Sciences

19 ul. Pawinskiego 5A

20 02-106 Warsaw

21 Poland 
22

23

\section{Abstract}

25 26 27 28 29

Email: szczesny@ibb.waw.pl

Recent addition to the repertoire of gene expression regulatory mechanisms are polyadenylate (polyA) tracks encoding for poly-lysine runs in protein sequences. Such tracks stall translation apparatus and induce frameshifting independently of the effects of charged nascent poly-lysine sequence on the ribosome exit channel. As such they substantially influence the stability of mRNA and amount of protein produced from a given transcript. Single base changes in these regions are enough to exert a measurable response on both protein and mRNA abundance, and makes each of these sequences potentially interesting case studies for effects of synonymous mutation, gene dosage balance and natural frameshifting. Here we present the PATACSDB, a resource that contain comprehensive list of polyA tracks from over 250 eukaryotic genomes. Our data is based on Ensembl genomic database of coding sequences and filtered with algorithm of 12A-1 which selects sequences of polyA tracks with a minimal length of $12 \mathrm{~A}$ 's allowing for one mismatched base. The PATACSDB database is accesible at: http://sysbio.ibb.waw.pl/patacsdb. Source code is available for download from GitHub repository at http://github.com/habich/PATACSDB, including the scripts to recreate the database from the scratch on user's own computer.

\section{Background}

1 The classical view of the genetic information flow inside living cells, that is transcription from 2 DNA to RNA and finally translation of mRNA into protein, is a subject of continous 
43 modification for both, direction of the flow and the number of players involved. Over decades of

44 research we keep accumulating evidences of several control points at different levels of these

45 processes. The past studies were focused on transcriptional regulation, but more recently 46 regulation of gene expression at the level of translation drew researchers' attention. Translational 47 regulation generally controls the amount of protein synthesised from a given mRNA through 48 several mechanisms, targeting recruitment of ribosomes to the transcript, elongation speed, 49 termination and as a proxy to all these processes mRNA stability. Ribosome stalling, that is 50 pausing of ribosome during translational cycle, is recognized by components of several mRNA 51 surveillance pathways. As a result of impeded rate of ribosome along the mRNA, the transcript is 52 endonucleolytically cleaved and nascent albeit incomplete protein product is degraded by 53 proteasome (Shoemaker \& Green, 2012). Over the years we have got to know that certain 54 sequence features can trigger ribosome stalling. These are damaged bases (Cruz-Vera et al., 55 2004), stable stem-loop structures (Doma \& Parker, 2006), rare codons (Letzring, Dean \& 56 Grayhack, 2010), mRNAs lacking stop codons (so called non-stop mRNAs) (Dimitrova et al., 57 2009), runs of codons that encode consecutive basic aminoacids (Kuroha et al., 2010; Brandman 58 et al., 2012), or finally, runs of adenines encoding poly-lysine tracks (Koutmou et al., 2015; 59 Arthur et al., 2015).

60 We have recently shown that polyA tracks trigger a response in a different manner than runs of 61 basic aminoacids (Arthur et al., 2015). In addition to stalling, occasionally they lead to ribosome 62 sliding on mRNA transcript which results in production of additional frameshifted product next 63 to the known and well annotated gene protein product. As such polyA track sequences may 64 support programed translational frameshifts in such mRNA transcripts giving rise to alternative 65 protein products from those genes. This feature of polyA track genes resembles programmed 
66 frameshifting observed in viral genes with slippery sequences however without a need for

67 additional mRNA structures that induces ribosome stalling in known viral transcripts (Chen et

68 al., 2014; Yan et al., 2015). The ultimate control over the production and stability of alternative

69 transcripts from polyA track genes in Eukaryotes would be based on mRNA surveillance

70 mechanisms, mainly non-sense mediated mRNA decay (NMD) or if the kinetic stall persists by

71 no-go mRNA decay (NGD). PolyA tracks are highly conserved in genes among Eukaryotes and

72 it is likely that they represent a universal translational attenuators or programed translational

73 frameshift signals. Intrinsically this novel RNA motif plays an important role in balancing gene

74 dosage and homeostasis of cellular environment. The level of attenuation, frameshifting and

75 exact role of polyA tracks in organisms homeostasis is still to be elucidated.

\section{PATACSDB server}

77 While there are several resources devoted to polyadenylation signals in genomic sequences, 78 these have different sequence signature and refer to the processing of mRNA, not translation. No 79 genomic database reports polyA tracks in coding sequences, therefore we have designed 80 PATACSDB (PolyA Translational Attenuators in Coding Sequences DataBase), a resource 81 devoted to collection of such features among eukaryotic organisms. In concordance with our 82 experimental data from the controlled expression of reporter sequences or natural gene 83 expression profiles we have designed a 12A-1 pattern, that is pattern of twelve adenines in 84 coding region allowing for one mismatch. Based on our experiments, this is a minimal pattern 85 that should result in reduction of expression by roughly $30 \%$, a magnitude that can potentially

86 have a measurable biological impact in human cells (Arthur et al., 2015). We have extrapolated 87 this pattern to other organisms, because without further experimental work we have no way to 
88 define the minimal polyA pattern in other organisms. We have analyzed eukaryotic Ensembl 89 genomes (Flicek et al., 2014) for the presence of this pattern in coding sequences, using only 90 these entries for which coding sequence matched reported translated sequence. This was done 91 not only on standard Ensembl genomes but its additional eukaryotic databases like Ensembl 92 Protists and Ensembl Metazoa. As a result, we have identified 197964 genes in 254 genomes that 93 carry 446206 polyA tracks.

\section{PolyA tracks across eukaryotic organisms}

95 In the previous studies (Koutmou et al., 2015; Arthur et al., 2015) we focused mainly on polyA tracks from human and yeast genomes, using NCBI (Pruitt et al., 2014) database and SGD

97 (Cherry et al., 1998) as data sources, respectively. Overall there is a good agreement between our previous analysis and this study for high eukaryotes, while we see some discrepancies for lower eukaryotes, such as yeast. For example, in the previous study we have underestimated the number of polyA-carrying genes in yeast by an order of magnitude (29 vs 369 ) - a result of different data source.

102 The percentage of polyA carrying transcripts varies from organism to organism and exceeds $60 \%$ 103 for Plasmodium species, well known for their AT-rich genome (see Table 1 for summary). 104 However, the distribution of lengths of polyA tracks is quite similar across whole observed spectrum of AT-content (Fig.1). It might be that the single Plasmodium genus is skewing the distribution, as the species distribution of genomic databases is heavily biased. In human, around only around $1 \%$ of transcripts coming from ca. $2 \%$ of genes carry polyA track and as such, are subjects of translational attenuation. This is close to a median across all analyzed genomes.

109 Furthermore, we did not find any correlation between organismal complexity and number of 
110 polyA-affected genes. This might indicate that such feature is a constituent element of 111 translational machinery, unrelated to external factors and regulatory mechanisms.

\section{Software architecture}

113 The main table consists of protein common name, gene and transcripts Ensembl ids, location of

114 the polyA track expressed as percentage (allows for quick identification of cases where polyA 115 track is either at the end or at the beginning of the protein) and finally, the identified polyA track 116 with a context of surrounding sequence. All columns are sortable. By default, the table is sorted

117 by protein name, alphabetically. Sorting gene and transcript ids is also alphabetical. Location is 118 sorted numerically. The rows with polyA sequences is sortable by polyA track length, so the user 119 can quickly identify sequences with the longest track in particular organism. Obviously, due to 120 used pattern, the shortest polyA tracks have length of 12 nucleotides. To facilitate quick 121 interaction with tables, we have used Bootstrap-table library that allows for easy and intuitive 122 sorting and searching through all fields in particular genome.

Project was created using Python 2.7. To parse biological data we used Biopython 1.65.

124 To compare protein and cdna sequences we used local version of NCBI blast + software v. 125 2.2.31. To run the web service we used Flask v.0.10.1. We used SQLite3 database engine and 126 SQLAlchemy for database access. To query Ensembl database we used mysql client. We also 127 used two other Python libraries: xmltodict and requests. The most difficult task was to ensure 128 short page load times given the large dataset we worked on. To solve this problem we have 129 created additional tables in database which contain metadata with the heaviest queries. This 130 solution decreased time of loading more than 20 times. 
132 database and create our database with $12 \mathrm{~A}-1$ pattern. In the second step we use created database

133 to provide information to web service. This architecture allows to separate obtaining data and

134 running web service thus during analysis of new version of Ensembl data we still can provide

135 data about old version, and change between versions can be done in seconds without user

136 noticing. In the future we will work on parallelization process of Ensembl data analysis to

137 speedup first step. It is likely that polyA segments are not the only sequence determinants of

138 translation efficiency in coding sequences and further studies will discover more of such motifs

139 or different lengths of minimal polyA pattern for a particular organism. Design of the

140 PATACSDB engine allows for easy modification towards finding and cataloguing of novel

141 sequence patterns.

142

143

144

145 
147 Table 1. Summary of the content of PATACSDB

\begin{tabular}{|c|c|}
\hline Feature & Value \\
\hline Total number of polyA-carrying transcripts & 197964 \\
\hline $\begin{array}{l}\text { Highest percentage of polyA-carrying transcripts } \\
\text { (first 5) }\end{array}$ & $\begin{array}{l}\text { Plasmodium berghei } 68.259 \% \\
\text { Plasmodium yoelii } 17 \text { x } 64.957 \% \\
\text { Plasmodium falciparum } 63.539 \% \\
\text { Plasmodium chabaudi } 63.372 \% \\
\text { Plasmodium reichenowi } 62.933 \%\end{array}$ \\
\hline $\begin{array}{l}\text { Lowest percentage of polyA-carrying transcripts } \\
\text { (first 5) }\end{array}$ & $\begin{array}{l}\text { Pythium vexans } 0.025 \% \\
\text { Saprolegnia diclina vs } 200.038 \% \\
\text { Leishmania major } 0.048 \% \\
\text { Phytophthora sojae } 0.058 \% \\
\text { Salpingoeca rosetta } 0.060 \%\end{array}$ \\
\hline $\begin{array}{l}\text { Median and average percentage of polyA- } \\
\text { carrying transcripts }\end{array}$ & $2.0 \%$ and $7.6 \%$ respectively \\
\hline The longest polyA tracks (first 10) & $\begin{array}{l}132 \mathrm{nt} \text { - CDO62875 [Plasmodium reichenowi] } \\
131 \mathrm{nt} \text { - CDO63348 [Plasmodium reichenowi] } \\
111 \mathrm{nt}-\text { ETW31025 [Plasmodium falciparum } \\
\text { fch 4] } \\
109 \mathrm{nt}-\text { ETW57402 [Plasmodium falciparum } \\
\text { palo alto uganda] } \\
107 \mathrm{nt} \text { - ETW41820 [Plasmodium falciparum } \\
\text { nf135 } 5 \text { c10] } \\
107 \mathrm{nt}-\text { ETW15539 [Plasmodium falciparum } \\
\text { vietnam oak knoll fvo] } \\
97 \mathrm{nt}-\text { CDO66404 [Plasmodium reichenowi] } \\
95 \mathrm{nt}-\text { EUT78604 [Plasmodium falciparum } \\
\text { santa lucia] } \\
89 \mathrm{nt}-\text { ETW44841 [Plasmodium falciparum } \\
\text { nf135 } 5 \text { c10] } \\
88 \mathrm{nt}-\text { ETW48723 [Plasmodium falciparum } \\
\text { malips096 e11] }\end{array}$ \\
\hline
\end{tabular}

148

149

150

151 
152

153 Fig.1. Distribution of polyA lengths vs AT-ratio of analyzed genomes. Data for lengths of polyA were

154 divided into 16 bins distributed evenly across spectrum of AT-richness of analyzed genomes. Width of a

155 box is proportional to the number of observances in a particular bin. Lines denote $1.5^{*} \mathrm{IQR}$ (interquantile

156 range). Outliers were removed for clarity. Data start at length of 12 , as this was the length of the minimal 157 pattern used.

158

159

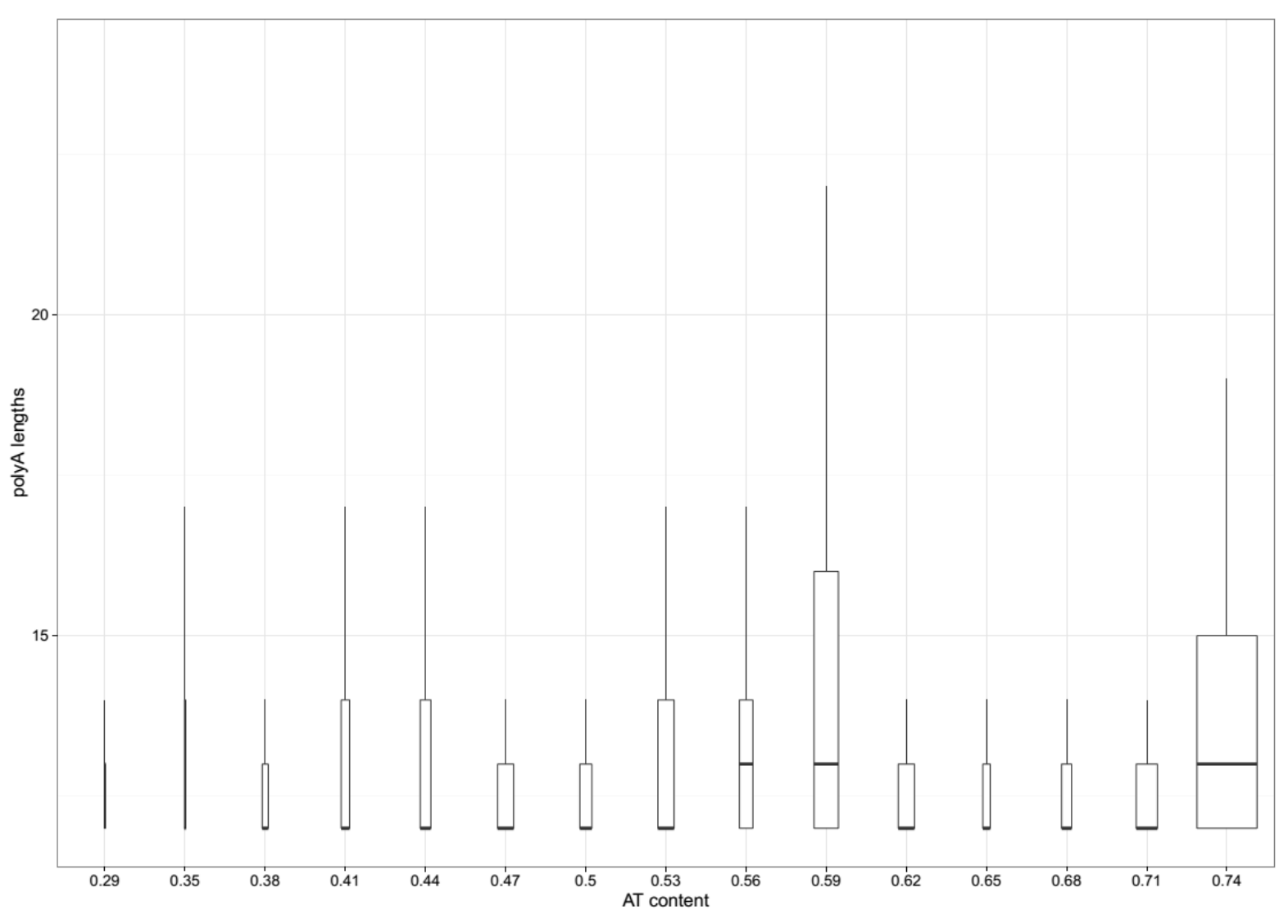

160 References

161 Arthur L., Pavlovic-Djuranovic S., Smith-Koutmou K., Green R., Szczesny P., Djuranovic S. 2015.

162 Translational control by lysine-encoding A-rich sequences. Science advances 1.

163 Brandman O., Stewart-Ornstein J., Wong D., Larson A., Williams CC., Li G-W., Zhou S., King D., Shen 
164

165

166

167

168

169

170

171

172

173

174

175

176

177

178

179

180

181

182

183

184

185

186

187

188

189

PS., Weibezahn J., Dunn JG., Rouskin S., Inada T., Frost A., Weissman JS. 2012. A ribosome-bound quality control complex triggers degradation of nascent peptides and signals translation stress. Cell 151:1042-1054.

Chen J., Petrov A., Johansson M., Tsai A., O’Leary SE., Puglisi J. 2014. Dynamic pathways of -1 translational frameshifting. Nature 512:328-332.

Cherry JM., Adler C., Ball C., Chervitz SA., Dwight SS., Hester ET., Jia Y., Juvik G., Roe T., Schroeder M., Others. 1998. SGD: Saccharomyces genome database. Nucleic acids research 26:73-79.

Cruz-Vera LR., Magos-Castro MA., Zamora-Romo E., Guarneros G. 2004. Ribosome stalling and peptidyl-tRNA drop-off during translational delay at AGA codons. Nucleic acids research 32:44624468.

Dimitrova LN., Kuroha K., Tatematsu T., Inada T. 2009. Nascent peptide-dependent translation arrest leads to Not4p-mediated protein degradation by the proteasome. The Journal of biological chemistry 284:10343-10352.

Doma MK., Parker R. 2006. Endonucleolytic cleavage of eukaryotic mRNAs with stalls in translation elongation. Nature 440:561-564.

Flicek P., Amode MR., Barrell D., Beal K., Billis K., Brent S., Carvalho-Silva D., Clapham P., Coates G., Fitzgerald S., Gil L., Girón CG., Gordon L., Hourlier T., Hunt S., Johnson N., Juettemann T., Kähäri AK., Keenan S., Kulesha E., Martin FJ., Maurel T., McLaren WM., Murphy DN., Nag R., Overduin B., Pignatelli M., Pritchard B., Pritchard E., Riat HS., Ruffier M., Sheppard D., Taylor K., Thormann A., Trevanion SJ., Vullo A., Wilder SP., Wilson M., Zadissa A., Aken BL., Birney E., Cunningham F., Harrow J., Herrero J., Hubbard TJP., Kinsella R., Muffato M., Parker A., Spudich G., Yates A., Zerbino DR., Searle SMJ. 2014. Ensembl 2014. Nucleic acids research 42:D749-55.

Koutmou KS., Schuller AP., Brunelle JL., Radhakrishnan A., Djuranovic S., Green R. 2015. Ribosomes slide on lysine-encoding homopolymeric A stretches. eLife 4.

Kuroha K., Akamatsu M., Dimitrova L., Ito T., Kato Y., Shirahige K., Inada T. 2010. Receptor for activated $\mathrm{C}$ kinase 1 stimulates nascent polypeptide-dependent translation arrest. EMBO reports 
190

191 192

193 194 195
11:956-961.

Letzring DP., Dean KM., Grayhack EJ. 2010. Control of translation efficiency in yeast by codonanticodon interactions. $R N A$ 16:2516-2528.

Pruitt KD., Brown GR., Hiatt SM., Thibaud-Nissen F., Astashyn A., Ermolaeva O., Farrell CM., Hart J., Landrum MJ., McGarvey KM., Murphy MR., O’Leary NA., Pujar S., Rajput B., Rangwala SH., Riddick LD., Shkeda A., Sun H., Tamez P., Tully RE., Wallin C., Webb D., Weber J., Wu W., DiCuccio M., Kitts P., Maglott DR., Murphy TD., Ostell JM. 2014. RefSeq: an update on mammalian reference sequences. Nucleic acids research 42:D756-763.

Shoemaker CJ., Green R. 2012. Translation drives mRNA quality control. Nature structural \& molecular biology 19:594-601.

Yan S., Wen J-D., Bustamante C., Tinoco I Jr. 2015. Ribosome excursions during mRNA translocation mediate broad branching of frameshift pathways. Cell 160:870-881. 